VoL. 45 (1992) [423-437]

\title{
SIMULTANEOUS MONOTONE APPROXIMATION IN LOW-ORDER MEAN
}

\author{
Robert Huotari and Salem Sahab
}

\begin{abstract}
Suppose that $f, g \in L_{\infty}[0,1]$ have discontinuities of the first kind only. Using the measure, $\max \left\{\|f-h\|_{p},\|g-h\|_{p}\right\}$, of simultaneous $L_{p}$ approximation, we show that the best simultaneous approximations, $h_{p}$, to $f$ and $g$ by nondecreasing functions converge uniformly as $p \rightarrow 1$. Part of the proof involves a discussion of discrete simultaneous approximation in a general context. We discuss the inheritance of properties of $f$ and $g$ by $h_{p}$, and of $h_{p}$ by $h_{1}$.
\end{abstract}

\section{INTRODUCTION}

A context which calls for simultaneous approximation is that of fitting a multivariate function by a univariate function. For example if $f: A \times B \rightarrow \mathbb{R}$, then the problem is to approximate the set of univariate functions $\mathcal{F}:=\left\{f\left(x, y_{0}\right): y_{0} \in B\right\}$ by a single function $g: A \rightarrow \mathbb{R}$. In the present paper we shall restrict our attention to the case where $\mathcal{F}$ consists of exactly two functions. In measuring the distance from $g$ to $\mathcal{F}$, two norms must be used; their composition is called a vectorial norm.

When one considers the continuum of normed linear spaces $\left\{L_{p}(\Omega, \Sigma, \mu): 1 \leqslant p \leqslant\right.$ $\infty\}$, three vectorial norms present themselves as being most natural for measuring simultaneous approximation as $p$ varies. The simultaneous $L_{p}$-distance from $f$ and $g$ to $h$ could be calculated by $\left(\|f-h\|_{p}^{p}+\|g-h\|_{p}^{p}\right)^{1 / p}$, by $\left(\|f-h\|_{p}+\|g-h\|_{p}\right)$, or by $\max \left(\|f-h\|_{p},\|g-h\|_{p}\right)$. In the first of these vectorial norms, the theory of simultaneous approximation is strongly related to that of single approximation on $L_{p} \times$ $L_{p}$, and has been extensively studied $[15,16,17]$. The second norm has not, to our knowledge, been widely studied vis-a-vis the continuum of $L_{p}$-spaces, and is the subject of a planned future work. The third norm seems most natural for studying the uniform, as it relates to the $L_{p}$, simultaneous approximation operator, $S_{p}$; this study was begun in [8]. It is the norm used in the classical theory of Chebyschev centres [18] and provides the context in which the simultaneous approximation problem (for any compact set of approximations) is most naturally stated. In the present paper we continue the study of

Received 14 May 1991

This research was partially supported by Grant \# 170-410 from King Abdulaziz University.

Copyright Clearance Centre, Inc. Serial-fee code: 0004-9729/92 \$A2.00+0.00. 
this "max norm" in $L_{p}$-simultaneous approximation, with primary focus on small values of $p$, and on convex discrete and monotone continuous approximation of functions on a probability space.

Most of the results to be presented here relate to the continuity of $S_{p}$; for $p$ fixed, and as $p$ varies. In [8], it was shown, for a large class of approximating sets in the discrete case, and for the approximating set $\mathcal{M}$ (nondecreasing functions on $[0,1]$ ) in the continuous case, that $S_{p}(f, g)$ converges as $p \rightarrow \infty$. In the present paper, we establish similar results for the case $p \rightarrow 1$. The existence of $\lim _{p \rightarrow 1} S_{p}(f, g)$ ameliorates the nonuniqueness of the 1-b.s.a. [10].

We begin with some definitions and notation. If $a, b \in \mathbb{R}$ (the set of all real numbers), let $a \vee b=\max (a, b)$ and $a \wedge b=\min (a, b)$. If $f, g: \mathbb{R} \rightarrow \mathbb{R}$, define $f \vee g$ by $(f \vee g)(t)=f(t) \vee g(t)$ and $f \wedge g$ by $(f \wedge g)(t)=f(t) \wedge g(t)$. Let $(\mathcal{X}, d)$ be a metric space. If $\mathcal{K} \subset \mathcal{X}$ and $f, g, h \in \mathcal{X}$, let $d(f, g ; h)=d(f, h) \vee d(g, h)$, let $d(f ; \mathcal{K})=\inf _{h \in \mathcal{K}}\{d(f, h)\}$, and let $d(f, g ; \mathcal{K})=\inf _{h \in \mathcal{K}}\{d(f, g ; h)\}$. We say that $h^{*} \in \mathcal{K}$ is a best (respectively, best simultaneous) d-approximation to $f$ (respectively, to $f$ and $g$ ) from $\mathcal{K}$ if $d\left(f, h^{*}\right)=d(f ; \mathcal{K})$ (respectively, $d\left(f, g ; h^{*}\right)=d(f, g ; \mathcal{K})$ ). In this case, we say that $h^{*}$ is a $d$-b.a. to $f$ (respectively, $d$-b.s.a. to $f$ and $g$ ). If there is a unique $d$-b.s.a. to $f$ and $g$ from $\mathcal{K}$, we denote it by $S(f, g)$. In subsequent sections of this paper we shall specialise by letting $\mathcal{X}=L_{p}$, but for the present we shall stay in a general context to state two theorems which we have not seen in the literature. The first relates to the continuity of $S$ and its proof is mutatis mutandis the same as that of (2.5) in [14].

Theorem 1. If $\mathcal{K}$ is compact in $(\mathcal{X}, d)$ and, for every $f, g \in \mathcal{X}, S(f, g)$ is uniquely defined, then, for any $\varepsilon>0$ there exists $\delta>0$ such that $d\left(S\left(f^{\prime}, g^{\prime}\right), S(f, g)\right)<$ $\varepsilon$ whenever $d\left(f, f^{\prime}\right)<\delta$ and $d\left(g, g^{\prime}\right)<\delta$.

Theorem 2. If $d$ is induced by a norm and if $h$ is a $d$-b.s.a. to $f$ and $g$ from $\mathcal{K}$ but not a $d$-b.a. to $f$ from $\mathcal{K}$, then $d(f, h) \leqslant d(g, h)$.

Proof: Suppose the theorem is false. Then $d(g, h)<d(f, h)$. Since $h$ is not a $d$-b.a. to $f$, there exists $f^{*} \in \mathcal{K}$ such that $d\left(f, f^{*}\right)<d(f, h)$. For $\alpha \in \mathbb{R}$, let $H(\alpha)=(1-\alpha) h+\alpha f^{*}$, let $G(\alpha)=d(g, H(\alpha))$, and let $F(\alpha)=d(f, H(\alpha))$. Since $d$ is induced by a norm, $G$ and $F$ are continuous. Thus, since $G(0)<F(0)$, there must be a $\beta>0$ such that $G(\beta)<F(\beta)$. Since $F$ is convex and since $F(1)<F(0)$, $F(\beta)<F(0)$. Thus $G(\beta)<F(0)$. Let $h^{*}=H(\beta)$. By the last two inequalities,

$$
d\left(f, g ; h^{*}\right)<d(f, h)=d(f, g ; h),
$$

which is a contradiction.

Let $h^{*}=S(f, g), f^{*}=S(f, f)$, and $g^{*}=S(g, g)$. In [3], it was shown that if $d$ is induced by an inner product, if $\mathcal{K}$ is a linear subspace, and if $f^{*} \neq h^{*} \neq g^{*}$, then $h^{*}$ 
must be of the form

$$
h^{*}=\lambda f^{*}+(1-\lambda) g^{*},
$$

where $\lambda \in(0,1)$ is determined by the equation

$$
d\left(f, h^{*}\right)=d\left(g, h^{*}\right) \text {. }
$$

If the requirement that $\mathcal{K}$ be a linear subspace is removed, then (i) doesn't hold in general even if we are in Hilbert space. To show this, let $f=\{3,0,5,0,7,0\}$, $g=\{-3,1,0,-2,1,-1\}, w=(1 / 15)\{1,2,1,4,1,6\}$, and let $\mathcal{K}=\mathcal{M}$, the closed convex cone of nondecreasing $n$-tuples in $\ell_{2}^{n}(w)$. Then $f_{2}=\{1,1,1,1,1,1\}, g_{2}=$ $(-1 / 7)\{-21,6,6,6,5,5\}$ and $h_{2}=\{0,1 / 4,1,1,1,1\}$. A simple calculation shows that $\left\|f-h_{2}\right\|_{2}^{2}=\left\|g-h_{2}\right\|_{2}^{2}=569 / 120$, but there does not exist a $\lambda \in(0,1)$ for which $h_{2}=\lambda f_{2}+(1-\lambda) g_{2}$.

However, in the more general context of Theorem (2), (ii) does hold, and is proven in the following corollary. Geometrically speaking, the corollary says that if the relative Chebyschev centre of $f$ and $g$ is a nearest point to neither $f$ nor $g$, then it is a relative "midpoint" of $f$ and $g$.

Corollary 3. Suppose $d$ is induced by a norm and $\mathcal{K}$ is any convex subset of $\mathcal{X}$. If $h$ is a d-b.s.a. to $f$ and $g$ from $\mathcal{K}$, but is a d-b.a. to neither $f$ nor $g$, then $d(f, h)=d(g, h)$.

Corollary (3) can be generalised to the simultaneous approximation of $n$ functions $f^{1}, \ldots, f^{n}$ as follows. If $1 \leqslant i<j \leqslant n$, if $h$ is a $d$-b.s.a. of $\left\{f^{1}, \ldots, f^{n}\right\}$, and if $h$ is a $d$-b.a. to neither $f^{i}$ nor $f^{j}$, then $d\left(f^{i}, h\right)=d\left(f^{j}, h\right)$. However, in some of the results stated below, we assume in an essential way that $n=2$.

In the remainder of this paper we shall assume that $\mathcal{X}=L_{p}(\Omega, \Sigma, \mu)$ (where $(\Omega, \Sigma, \mu)$ is a probability space and $1 \leqslant p \leqslant \infty)$, that $\mathcal{K}$ is an $\|\cdot\|_{1}$-closed convex subset of $\mathcal{X}$, and that $f, g \in L_{\infty}$. Let $d_{p}$ be the metric induced by $\|\cdot\|_{p}$ and let $p$-b.s.a. and $p$-b.a. denote $d_{p}$-b.s.a. and $d_{p}$-b.a., respectively. For $1 \leqslant p \leqslant \infty$, let $\mu_{p}(f, g ; \mathcal{K})$ consist of every $p$-b.s.a. to $f$ and $g$ from $\mathcal{K}$. If $1<p<\infty$, then $\mu_{p}(f, g ; \mathcal{K})$ is a singleton [3], which we denote by $S_{p}(f, g)$ or by $h_{p}$. We denote $S_{p}(f, f)$ by $f_{p}$.

\section{Discrete Simultaneous Approximation}

In this section we assume that $\Omega=\{1,2, \ldots, n\}$, that $\Sigma=2^{n}$, that $\mu(\{i\})=$ $w_{i}>0$ (where $\sum_{i=1}^{n} w_{i}=1$ ), and that $\mathcal{K}$ is any $\|\cdot\|_{1}$-closed convex subset of $\mathcal{X}=\mathbb{R}^{n}$. The underlying norm is the weighted $\ell_{p}$ norm, defined by $\|h\|_{p}=\left(\sum_{i=1}^{n} w_{i}|h(i)|^{p}\right)^{1 / p}$, for $1 \leqslant p<\infty$, and $\|h\|_{\infty}=\max _{1 \leqslant i \leqslant n}\left(w_{i}|h(i)|\right)$.

We begin with a lemma that will be used in compactness arguments. 
Lemma 4. The set $\mathcal{H}=\left\{h_{p}: 1<p<\infty\right\}$ is uniformly bounded. Thus, every sequence in $\mathcal{H}$ has a convergent subsequence.

Proof: Let $z \in \mathcal{K}$ be fixed. For any $p \in(1, \infty),\left\|h_{p}\right\|_{p}-\|f\|_{p} \leqslant\left\|h_{p}-f\right\|_{p} \leqslant$ $d_{p}\left(f, g ; h_{p}\right) \leqslant d_{p}(f, g ; z) \leqslant d_{\infty}(f, g ; z)$ so $\left\|h_{p}\right\|_{p} \leqslant A:=\|f\|_{\infty}+d_{\infty}(f, g ; z)$ and, for $1 \leqslant i \leqslant n, w_{i}\left|h_{p}(i)\right|^{p} \leqslant A^{p}$. Since $w_{i} \leqslant 1$ and $p>1, w_{i}\left|h_{p}(i)\right| \leqslant w_{i}^{1 / p}\left|h_{p}(i)\right| \leqslant A$, so

$$
\left\|h_{p}\right\|_{\infty} \leqslant A \max \left\{w_{i}^{-1}: 1 \leqslant i \leqslant n\right\}
$$

The second assertion follows from the fact that every bounded sequence in $\mathbb{R}^{n}$ has a convergent subsequence.

One of our primary concerns is the continuity of $h_{p}$ as a function of $p$. The following theorem establishes this continuity on the interval $(1, \infty)$.

Theorem 5. The function $\Pi:((1, \infty), \mid \cdot) \rightarrow\left(\mathbb{R}^{n},\|\cdot\|_{\infty}\right)$ defined by $\Pi(p)=h_{p}$ is continuous.

ProOF: If the theorem is false, then there exist $p \in(1, \infty)$ and $p_{k} \rightarrow p$ such that $\lim _{k \rightarrow \infty}\left\|h_{p_{k}}-h_{p}\right\|_{\infty} \neq 0$. By (4), $\left\{h_{p_{k}}\right\}$ has a subsequence $\left\{h_{q_{k}}\right\}$ which converges to an element $h^{*} \neq h_{p}$. We now show that, to the contrary, it must be that $h^{*}=h_{p}$.

Let $\varepsilon>0$ be given. Since $\lim _{k \rightarrow \infty}\|z\|_{q_{k}}=\|z\|_{p}$ for every $z \in \mathbb{R}^{n}$, there exists $N_{1}$ such that for every $k \geqslant N_{1}$ and for $z=f, g$,

$$
\left\|z-h_{p}\right\|_{p}-\varepsilon<\left\|z-h_{p}\right\|_{q_{k}}<\left\|z-h_{p}\right\|_{p}+\varepsilon
$$

By the definition of best simultaneous approximation, $d_{q_{k}}\left(f, g ; h_{q_{k}}\right) \leqslant d_{q_{k}}\left(f, g ; h_{p}\right)$ so, for every $k \geqslant N_{1}$,

$$
d_{q_{k}}\left(f, g ; h_{q_{k}}\right) \leqslant d_{p}\left(f, g ; h_{p}\right)+\varepsilon .
$$

By our assumption, there exists $N_{2}$ such that, for every $k \geqslant N_{2},\left\|h_{q_{k}}-h^{*}\right\|_{\infty}<\varepsilon$ and, for $z=f, g$,

$$
\begin{aligned}
\left\|z-h^{*}\right\|_{q_{k}} & \leqslant\left\|z-h_{q_{k}}\right\|_{q_{k}}+\left\|h_{q_{k}}-h^{*}\right\|_{q_{k}} \\
& \leqslant\left\|z-h_{q_{k}}\right\|_{q_{k}}+\eta\left\|h_{q_{k}}-h^{*}\right\|_{\infty} \\
& <\left\|z-h_{q_{k}}\right\|_{q_{k}}+\varepsilon .
\end{aligned}
$$

Let $N=N_{1} \vee N_{2}$. By (i) and (ii), for every $k \geqslant N$,

$$
d_{q_{k}}\left(f, g ; h^{*}\right)<d_{q_{k}}\left(f, g ; h_{q_{k}}\right)+\varepsilon \leqslant d_{p}\left(f, g ; h_{p}\right)+2 \varepsilon
$$


which implies that $d_{p}\left(f, g ; h^{*}\right)<d_{p}\left(f, g ; h_{p}\right)+2 \varepsilon$. Since $\varepsilon$ is arbitrary and since $h_{p}$ is the unique $p$-b.s.a. to $f$ and $g$, it must be that $h^{*}=h_{p}$.

The following corollary is also related to continuity, but includes the endpoints, 1 and $\infty$. Its proof uses the continuity of $\|z\|_{p}$ as a function of $p$ and the definition of $d_{p}(f, g ; \mathcal{K})$.

Corollary 6. The function $D$, defined by $D(p)=d_{p}(f, g ; \mathcal{K})$, is continuous on $[1, \infty]$.

The following technical lemma will be used in the proof that $h_{p}$ converges as $p \downarrow 1$.

LEMma 7. Either (i) $\|g-h\|_{1} \leqslant\|f-h\|_{1}$ for every $h$ in $\mu_{1}(f, g ; \mathcal{K})$ or (ii) $\|f-h\|_{1} \leqslant\|g-h\|_{1}$ for every $h$ in $\mu_{1}(f, g ; \mathcal{K})$.

Proof: Suppose $h^{\prime}, h^{\prime \prime} \in \mu_{1}(f, g ; \mathcal{K}),\left\|g-h^{\prime}\right\|_{1}<\left\|f-h^{\prime}\right\|_{1}$ and $\left\|g-h^{\prime \prime}\right\|_{1}>$ $\left\|f-h^{\prime \prime}\right\|_{1}$. Let $h^{*}=\left(h^{\prime}+h^{\prime \prime}\right) / 2$. Then

$$
\begin{aligned}
d_{1}\left(f, g ; h^{*}\right) & =\left\|f-\left(h^{\prime}+h^{\prime \prime}\right) / 2\right\|_{1} \vee\left\|g-\left(h^{\prime}+h^{\prime \prime}\right) / 2\right\|_{1} \\
& \leqslant \frac{1}{2}\left[\left(\left\|f-h^{\prime}\right\|_{1}+\left\|f-h^{\prime \prime}\right\|_{1}\right) \vee\left(\left\|g-h^{\prime}\right\|_{1}+\left\|g-h^{\prime \prime}\right\|_{1}\right)\right] \\
& <\frac{1}{2}\left[\left(\left\|f-h^{\prime}\right\|_{1}+\left\|g-h^{\prime \prime}\right\|_{1}\right) \vee\left(\left\|f-h^{\prime}\right\|_{1}+\left\|g-h^{\prime \prime}\right\|_{1}\right)\right] \\
& \leqslant \frac{1}{2}\left[\left(2\left\|f-h^{\prime}\right\|_{1}\right) \vee\left(2\left\|g-h^{\prime \prime}\right\|_{1}\right)\right] \\
& =d_{1}\left(f, g ; h^{\prime}\right),
\end{aligned}
$$

a contradiction.

The proof of the following theorem is modelled after the proof of [10, Theorem 2]. Throughout the demonstration, we shall assume without loss of generality that (7i) holds. For $1 \leqslant i \leqslant n$ define $\lambda_{i}: \mathbb{R}^{n} \rightarrow \mathbb{R}$ by $\lambda_{i}(h)=h(i)-f(i)$. Let $\mathcal{K}_{1}=\mu_{1}(f, g ; \mathcal{K})$. Clearly $\mathcal{K}_{1}$ is convex. We claim that

$$
\lambda_{i} \text { does not change sign on } \mathcal{K}_{1} .
$$

Indeed, for $x, y \in \mathcal{K}_{1}$ and $1 \leqslant i \leqslant n$, let $s=x-f$ and $t=y-f$. If $s(i)=a>0$ and $t(i)=-b<0$, let $z=(b s+a t) /(a+b)$. Then $z(i)=0$ and, for $k \neq i,|z(k)| \leqslant$ $(b|s(k)|+a|t(k)|) /(a+b)$, so

$$
\|z\|_{1}<\left(b\|s\|_{1}+a\|t\|_{1}\right) /(a+b)=\|s\|_{1} .
$$

Let $x^{*}=(b x+a y) /(a+b)$. Since $\mathcal{K}_{1}$ is convex, $x^{*} \in \mathcal{K}$. By the last inequality, $\left\|x^{*}-f\right\|_{1}<\|x-f\|_{1}$, so $d_{1}\left(f, g ; x^{*}\right)=\left\|x^{*}-f\right\|_{1}<\|x-f\|_{1}=d_{1}(f, g ; x)$. This proves $(*)$. 
Define $\gamma: \mathbb{R} \rightarrow \mathbb{R}$ by

$$
\gamma(r)= \begin{cases}|r| \ln |r|, & r \neq 0 \\ 0, & r=0\end{cases}
$$

For every $h \in \mathcal{K}$ and $1 \leqslant p<\infty$, let $F_{h}(p)=\|h-f\|_{p}^{p}$ and let

$$
\Gamma(h)=F_{h}^{\prime}(1)=\sum_{i=1}^{n}|h(i)-f(i)| \ln |h(i)-f(i)|
$$

Since $\gamma$ is strictly convex on $[0, \infty),(*)$ implies that $\Gamma$ is strictly convex on $\mathcal{K}_{1}$ and so has a unique minimiser; call it $h_{1}$.

In view of (4), to show that $\lim _{p \downarrow 1} h_{p}$ exists, it will suffice to exhibit a vector $h$ such that, for every sequence $\left\{p_{k}\right\} \downarrow 1, \lim _{k \rightarrow \infty} h_{p_{k}}=h$. The following lemma is a first step in this exhibition.

LEMMA 8. If $\left\{p_{k}: k \in \mathbb{N}\right\} \subset(1, \infty)$, if $p_{k} \downarrow 1$ and if $\left\|f-h_{p_{k}}\right\|_{p_{k}} \geqslant\left\|g-h_{p_{k}}\right\|_{p_{k}}$ for every $k \in \mathbb{N}$, then $\lim _{k \rightarrow \infty} h_{p_{k}}=h_{1}$.

PRoOF: If the lemma is false, then, by (4), there exists a sequence $\left\{q_{k}\right\} \subset\left\{p_{k}\right\}$ such that $q_{k} \downarrow 1$ and $h_{q_{k}} \rightarrow z \neq h_{1}$. Then

$$
\Gamma(z)>\Gamma\left(h_{1}\right)
$$

If $r \geqslant 0$, then the function $p \mapsto r^{p}$ is a convex function so the Mean Value Theorem implies that, for every $p>1, r \ln r \leqslant\left(r^{p}-r\right) /(p-1)$. Hence

$$
\Gamma\left(h_{q_{k}}\right) \leqslant \frac{1}{q_{k}-1} \sum_{i=1}^{n}\left\{\left|h_{q_{i}}(i)-f(i)\right|^{q_{k}}-\left|h_{q_{k}}(i)-f(i)\right|\right\}
$$

Since $h_{q_{k}}$ is a $q_{k}$-b.s.a. to $f$ and $g$ from $\mathcal{K}$, we have $\left\|f-h_{q_{k}}\right\|_{q_{k}} \leqslant d_{q_{k}}\left(f, g ; h_{q_{k}}\right) \leqslant$ $d_{q_{k}}\left(f, g ; h_{1}\right)$. This, along with (7i) gives

$$
\left\|f-h_{q_{k}}\right\|_{q_{k}} \leqslant\left\|f-h_{1}\right\|_{q_{k}}
$$

Since $h_{1} \in \mathcal{K}_{1}$ and (7i) holds, $\left\|f-h_{1}\right\|_{1}=d_{1}\left(f, g ; h_{1}\right) \leqslant d_{1}\left(f, g ; h_{q_{k}}\right)$. Since $\|z\|_{p}$ is a nondecreasing function of $p$ for every $z$ in $\mathbb{R}^{n},\left\|f-h_{1}\right\|_{1} \leqslant d_{q_{k}}\left(f, g ; h_{q_{k}}\right)=$ $\left\|f-h_{q_{k}}\right\|_{q_{k}}$, that is,

$$
\left\|f-h_{1}\right\|_{1} \leqslant\left\|f-h_{q_{k}}\right\|_{q_{k}} .
$$


By (ii), (iii), and (iv),

$$
\Gamma\left(h_{q_{k}}\right) \leqslant \frac{1}{q_{k}-1} \sum_{i=1}^{n}\left\{\left|h_{1}(i)-f(i)\right|^{q_{k}}-\left|h_{1}(i)-f(i)\right|\right\} .
$$

As $k \rightarrow \infty$, the right hand side of (v) approaches $\Gamma\left(h_{1}\right)$ so $\Gamma(z) \leqslant \Gamma\left(h_{1}\right)$, which contradicts (i), and proves the lemma.

THEOREM 9. The net $\left\{h_{p}: p>1\right\}$ converges as $p \downarrow 1$

PROoF: Suppose first that there is an $\alpha>1$ such that

$$
\left\|f-h_{p}\right\|_{p}<\left\|g-h_{p}\right\|_{p}, \quad p \in(1, \alpha) .
$$

In this case, if $p_{k} \downarrow 1$, then, without loss of generality, $\left\|f-h_{p_{k}}\right\|_{p_{k}}<\left\|g-h_{p_{k}}\right\|_{p_{k}}$ for every $k \in \mathbb{N}$, so (2) implies that $h_{p_{k}}=g_{p_{k}}$ and, by [10], $h_{p_{k}} \rightarrow g_{1}$, the natural best $\ell_{1}$-approximation to $g$ from $\mathcal{K}$, and the proof is complete.

Suppose (i) does not hold. Then there exists a sequence $\left\{p_{k}\right\}$ which satisfies the condition in Lemma 8, namely, $p_{k} \downarrow 1$ and $\left\|f-h_{p_{k}}\right\|_{p_{k}} \geqslant\left\|g-h_{p_{k}}\right\|_{p_{k}}$ for every $k \in \mathbb{N}$. If $q_{k} \downarrow 1$ and $\left\|f-h_{q_{k}}\right\|_{q_{k}}<\left\|g-h_{q_{k}}\right\|_{q_{k}}$, let $r_{k}=\sup \left\{p<q_{k}:\left\|f-h_{p}\right\|_{p} \geqslant\right.$ $\left.\left\|g-h_{p}\right\|_{p}\right\}$. We may assume without loss of generality that $\left\{r_{k}\right\} \subset\left\{p_{k}\right\}$. Then, by (5), $r_{k}<q_{k}$. By the Intermediate Value Theorem, $\left\|f-h_{p}\right\|_{p}<\left\|g-h_{p}\right\|_{p}$ for every $p$ in $\left(r_{k}, q_{k}\right)$, and, by (2), $h_{p}=g_{p}$ for every $p \in\left(r_{k}, q_{k}\right)$. Thus (5) implies that $\lim _{p \downarrow r_{k}} g_{p}=\lim _{p \downarrow r_{k}} h_{p}=h_{r_{k}}$. Since $G(p)=g_{p}$ is continuous on $(1, \infty)$ (the proof is similar to that of (5)), it must be that $h_{r_{k}}=g_{r_{k}}$. From the above considerations, we know that $h_{q_{k}} \rightarrow g_{1}$ and $h_{r_{k}} \rightarrow h_{1}$. But $h_{r_{k}}=g_{r_{k}} \rightarrow h_{1}$ so $h_{q_{k}} \rightarrow h_{1}$.

Thus, if (i) does not hold and if $q_{k} \downarrow 1$, then, without loss of generality, either $\left\{q_{k}\right\}=\left\{r_{k}\right\}$ or $\left\{q_{k}\right\}=\left\{r_{k}\right\} \cup\left\{s_{k}\right\}$, where, for every $k \in \mathbb{N},\left\|f_{r_{k}}-h_{r_{k}}\right\|_{r_{k}} \geqslant$

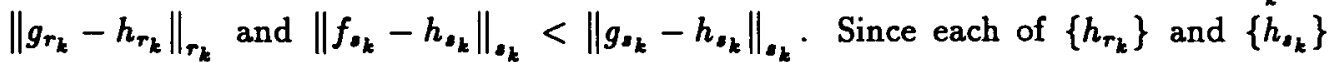
converges to $h_{1}$, so do $\left\{h_{q_{k}}\right\}$ and the net $\left\{h_{p}: p>1\right\}$. However, $\sup (f, g ; \mathcal{M})=\bar{h}=$ $\chi_{[0,1 / 2]}+2 \chi_{(1 / 2,1]}$ and $\inf (f, g ; \mathcal{M})=\underline{h}=\chi_{(1 / 2,1]}$ are not in $\mu_{1}(f, g ; \mathcal{M})$.

Combining (6) and (9), we have the following.

Corollary 10 . The set $\mu_{1}(f, g ; \mathcal{K})$ is nonempty.

\section{Simultaneous Monotone $L_{p}$-Approximation, $p \in[1, \infty]$}

In this section we shall assume that $\Omega=[0,1]$, that $\Sigma$ consists of all Lebesgue measurable subsets of $\Omega$, and that $\mu$ is Lebesgue measure. Let $\mathcal{K}=\mathcal{M}$, the set of all nondecreasing extended real-valued functions on $\Omega$ and let $f, g \in L_{\infty}$ have at most discontinuities of the first kind. Let $M=\|f\|_{\infty} \vee\|g\|_{\infty}$. 
LemMa 11. The set $\cup_{p=1}^{\infty} \mu_{p}(f, g ; \mathcal{M})$ is uniformly bounded by $M$.

Proof: If $h \in \mu_{p}(f, g ; \mathcal{M})$ but there is a $t \in(0,1)$ such that $h(t)>M$, then there is an $s \in(0,1)$ such that, for every $r>s, h(r)>M$. Let $h^{*}=h \wedge M$. Then $h^{*} \in \mathcal{M}$ and $d_{p}\left(f, g ; h^{*}\right)<d_{p}(f, g ; h)$, a contradiction. The case $\min h(t)<-M$ is treated similarly.

Lemma 12. If $1<p<\infty$ and $\mathcal{H} \subset \mathcal{M}$ is uniformly bounded by $B$, then there exist $h^{k} \in \mathcal{H}$ and $h \in \mathcal{M}$ such that $\|h\|_{\infty} \leqslant B$ and $\lim _{k \rightarrow \infty}\left\|h-h_{k}\right\|_{p}=0$.

Proof: By Helly's Theorem [12], there exist $h^{k} \in \mathcal{H}$ and $h \in \mathcal{M}$ such that $\|h\|_{\infty} \leqslant B$ and $h^{k} \rightarrow h$ pointwise on $\Omega$. Thus, by the Lebesgue Dominated Convergence Theorem, $\left\{h^{i}\right\}$ converges to $h$ in $L_{p}$.

In view of (11) we may, and will, assume that $\mathcal{M}$ consists of all nondecreasing functions $h$ such that $\|h\|_{\infty} \leqslant 2 M$. Thus, by (12), $\mathcal{M}$ is a compact subset of $L_{p}$ for $1<p<\infty$. By (1), $S_{p}$ is a $\|\cdot\|_{p}$-continuous function of $f$ and $g$. By a proof similar to that of (5), the following result can be obtained. If $q \in(1, \infty)$ then the function $\Pi:((1, q],|\cdot|) \rightarrow L_{q}$ defined by $\Pi(p)=h_{p}$ is a continuous function of $p$.

We now undertake to show that $\lim _{p \downarrow 1} h_{p}$ exists, so that the last result can be extended to $[1, q]$.

THEOREM 13. The net $\left\{h_{p}\right\}$ converges uniformly as $p \downarrow 1$.

PROOF: The length of the proof, and the fact that some of its waystations are of independent interest, warrant its division into several lemmas. We begin by showing that $S_{p}$ is a monotone operator.

Lemma (i) Suppose that $f^{i}, g^{i} \in L_{p}, i=1,2,1<p<\infty$. If $f^{1} \leqslant f^{2}$ and $g^{1} \leqslant g^{2}$, then $S_{p} f^{1} g^{1} \leqslant S_{p} f^{2} g^{2}$.

Proof: Let $h^{i}=S_{p} f^{i} g^{i}, i=1,2, T_{1}=h^{1} \wedge h^{2}$ and $T_{2}=h^{1} \vee h^{2}$; let $a_{i}=$ $\left|f^{i}-h^{i}\right|, b_{i}=\left|g^{i}-h^{i}\right|, c_{i}=\left|f^{i}-T_{i}\right|$ and $d_{i}=\left|g^{i}-T_{i}\right|, i=1,2$. By [11, Lemma 2],

so

$$
\begin{array}{ll}
a_{2}^{p}+a_{1}^{p} \geqslant c_{2}^{p}+c_{1}^{p} & \text { and } \quad b_{2}^{p}+b_{1}^{p} \geqslant d_{2}^{p}+d_{1}^{p}, \\
a_{2}^{p} \vee b_{2}^{p} \geqslant c_{2}^{p} \vee d_{2}^{p} & \text { or } \quad a_{1}^{p} \vee b_{1}^{p} \geqslant c_{1}^{p} \vee d_{1}^{p} .
\end{array}
$$

If the first case holds, then upon integrating, we obtain

$$
\left\|f^{2}-h^{2}\right\|_{p} \vee\left\|g^{2}-h^{2}\right\|_{p} \geqslant\left\|f^{2}-T_{2}\right\|_{p} \vee\left\|g^{2}-T_{2}\right\|_{p} .
$$

Since $S_{p} f^{2} g^{2}$ is uniquely defined, $h^{2}=T_{2} \geqslant h^{1}$. By similar reasoning, if the second case holds, then $h^{1}=T_{1} \leqslant h^{2}$. This completes the proof of (i). 
Lemma (ii) For $1<p<\infty$ and $c \in \mathbb{R}, S_{p}(f+c, g+c)=h_{p}+c$.

Proof: By the definition of $h_{p}$, we have for all $h \in K$

$$
\left\|f-h_{p}\right\|_{p} \vee\left\|g-h_{p}\right\|_{p} \leqslant\|f-h\|_{p} \vee\|g-h\|_{p} .
$$

For any $k \in K$, there exists $h \in K$ such that $h+c=k$, so

$$
\begin{aligned}
\left\|f+c-\left(h_{p}+c\right)\right\|_{p} \vee\left\|g+c-\left(h_{p}+c\right)\right\|_{p} & \leqslant\|f+c-(h+c)\|_{p} \vee\|g+c-(h+c)\|_{p} \\
& =\|f+c-k\|_{p} \vee\|g+c-k\|_{p} .
\end{aligned}
$$

This concludes the proof of (ii).

Lemma (iii) If $1<p<\infty$, if $I$ is an open interval, and if both $f$ and $g$ are constant on $I$, then $S_{p}(f, g)$ is constant on $I$.

Proof: Let $h=S_{p}(f, g)$, and let $\left.h^{\prime}\right|_{I}=-h+g+f$, and $\left.h^{\prime}\right|_{\Omega \backslash I}=h$. Note that $\left.h^{\prime}\right|_{I}$ is nondecreasing. For notational convenience, we let $\|k-l\|=\left(\int_{I}|k-l|^{p}\right)^{1 / p}$. Then $\|f-h\|=\left\|g-h^{\prime}\right\|$ and $\|g-h\|=\left\|f-h^{\prime}\right\|$. If $h^{\prime \prime}=2^{-1}\left(h+h^{\prime}\right)$ and $d=$ $2^{-1}(\|f-h\|+\|g-h\|)$, then both $\left\|f-h^{\prime \prime}\right\| \leqslant d$ and $\left\|g-h^{\prime \prime}\right\| \leqslant d$. But this implies that

$$
\left\|f-h^{\prime}\right\| \vee\left\|g-h^{\prime \prime}\right\| \leqslant\|f-h\| \vee\|g-h\| .
$$

Since $h^{\prime \prime}=(g+f) / 2$ is constant on $I$ and $h=S_{p}(f, g)$ it must be that $h^{\prime \prime}=h$ so $h^{\prime}=h$. Thus $\left.h\right|_{I}$ is both nondecreasing and nonincreasing, hence constant. This concludes the proof of (iii).

Since $f$ and $g$ have at most discontinuities of the first kind, they can be uniformly approximated by step functions (see [19]). Thus, for any $n \in \mathbb{N}$ there are step functions

$$
f^{n}=a_{1} \chi_{\left[0, t_{1}\right]}+\sum_{i=2}^{k_{n}} a_{i} \chi_{\left(t_{i-1}, t_{i}\right]},
$$

and

$$
g^{n}=b_{1} \chi_{\left[0, t_{1}\right]}+\sum_{i=2}^{k_{n}} b_{1} \chi_{\left(t_{i-1}, t_{i}\right]},
$$

(where $\chi_{A}$ is the indicator function of $A$, that is, $\chi_{A}(t)=1$ if $t \in A$ and $\chi_{A}(t)=0$ if $t \notin A$ ) such that $\left\|f-f^{n}\right\|_{\infty}<n^{-1}$ and $\left\|g-g^{n}\right\|_{\infty}<n^{-1}$, where $\left\{0=t_{0}<t_{1}<\right.$ $\left.\ldots<t_{n}=1\right\}$ is the common refinement of the partitions of $[0,1]$ associated with the canonical representations of $f^{n}$ and $g^{n}$. Let $h_{p}^{n}=S_{p}\left(f_{n}, g_{n}\right)$. By the last lemma, $h_{p}^{n}$ must have the form

$$
h_{p}^{n}=c_{1}^{p} \chi_{\left[0, t_{1}\right]}+\sum_{i=2}^{k_{n}} c_{i}^{p} \chi_{\left(t_{i-1}, t_{i}\right]} .
$$


Thus, we are in the context of weighted discrete simultaneous approximation (where $f^{n}=\left\{a_{i}\right\}_{i=1}^{k_{n}}, g^{n}=\left\{b_{i}\right\}_{i=1}^{k_{n}}, h_{p}^{n}=\left\{c_{i}^{p}\right\}_{i=1}^{k_{n}}$ and $\left.w_{i}=t_{i}-t_{i-1}\right)$ so, by (9), there are numbers $c_{i}^{1}, 1 \leqslant i \leqslant k_{n}$, such that

$$
\lim _{p \downarrow 1} h_{p}^{n}=h_{1}^{n}=c_{1}^{1} \chi_{\left[0, t_{1}\right]}+\sum_{i=2}^{k_{n}} c_{i}^{1} \chi_{\left(t_{i-1}, t_{i}\right]} .
$$

LemMA (viii) Let $f^{n}, g^{n}, h_{p}$ and $h_{p}^{n}$ be as defined above. Let $h_{p}$ be the best $L_{p}$-simultaneous approximation to $f$ and $g$ from $\mathcal{M}$. Then for every $\varepsilon>0$, there exists an $N=N(f, g, \varepsilon)$ such that for all $n \geqslant N$ and $p \in(1, \infty),\left\|h_{p}^{n}-h_{p}\right\|_{\infty}<\varepsilon$.

Proof: Let $\varepsilon>0$ be given. Then there is an integer $N \geqslant 1$ such that $\left\|f-f^{n}\right\|_{\infty}<\varepsilon$ and $\left\|g-g^{n}\right\|_{\infty}<\varepsilon$ for all $n \geqslant N$. Thus, except on a set of measure zero, $n \geqslant N$ implies that

$$
f^{n}<f+\varepsilon, \quad g^{n}<g+\varepsilon
$$

and

$$
f<f^{n}+\varepsilon, \quad g<g^{n}+\varepsilon .
$$

Applying (i) and (ii) to (ix) and (x) respectively, we obtain

$$
h_{p}^{n}<h_{p}+\varepsilon, \quad \text { and } \quad h_{p}<h_{p}^{n}+\varepsilon \text {, }
$$

which implies that $\left\|h_{p}^{n}-h_{p}\right\|_{\infty}<\varepsilon$.

We are now in a position to complete the proof of Theorem 13. Let $\varepsilon>0$ be given. Then there exists $N \geqslant 1$ such that $\left\|f^{n}-f^{m}\right\|_{\infty}<\varepsilon$, and $\left\|g^{n}-g^{m}\right\|_{\infty}<\varepsilon$ for all $n, m \geqslant N$. An argument similar to that in the last proof shows that there exists an $N=N(f, g, \varepsilon)$ such that for every $n, m \geqslant N$ and $p \in(1, \infty), h_{p}^{n}<h_{p}^{m}+\varepsilon$ and $h_{p}^{m}<h_{p}^{n}+\varepsilon$. Letting $p \downarrow 1$, we obtain

$$
\left\|h_{1}^{n}-h_{1}^{m}\right\|_{\infty}<\varepsilon, \quad n, m \geqslant N .
$$

Hence $\left\{h_{1}^{n}: n=1,2, \ldots\right\}$ converges uniformly to, say, $h_{1}$. Since the values of $N$ in (viii) and (xi) are independent of $p,($ vii), (vii) and (xi) and the triangle inequality imply that $h_{p}$ converges uniformly to $h_{1}$ as $p \downarrow 1$. This concludes the proof of Theorem 13 .

Let $h_{1}=\lim _{p \downarrow 1} h_{p}$ and define $S_{1}(f, g):=h_{1}$. Applying a version of (6), we have that $h_{1} \in \mu_{1}(f, g ; \mathcal{M})$. This proves the following:

Corollary 14. The set $\mu_{1}(f, g ; \mathcal{M})$ is nonempty.

We end this section with a discussion of the inheritance of the continuity of $f$ and $g$ by $h_{p}$. The theorem below is presented in [8], but is included here also for self-containment. We refer the reader to [1] for the definition of approximate continuity. 
THEOREM 15. If $f$ and $g$ are approximately continuous and $p \in(1, \infty)$, then $h_{p}$ is continuous on $(0,1)$.

Proof: Suppose for contradiction that $h_{p}$ has a jump discontinuity at $a \in(0,1)$. We may assume without loss of generality that $g(a) \leqslant f(a)$.

We may approximate the above functions by step functions. Indeed, let $\sigma=g(a)$, $\tau=f(a), \lambda=h_{p}\left(a^{-}\right)=\lim _{t \uparrow a} h_{p}(t)$ and $\mu=h_{p}\left(a^{+}\right)$and suppose that $\alpha>0$. By Lemma 9 , there exists an $\eta \in[\lambda, \mu]$ and $\varepsilon=\varepsilon(\alpha)>0$ such that

$$
\begin{gathered}
\max \left\{\alpha\left(|\tau-\mu|^{p}+|\tau-\lambda|^{p}\right), \alpha\left(|\lambda-\sigma|^{p}+|\mu-\sigma|^{p}\right)\right\} \\
=\max \left\{2 \alpha|\tau-\eta|^{p}, 2 \alpha|\eta-\sigma|^{p}\right\}+\varepsilon .
\end{gathered}
$$

If $\alpha$ is replaced by a multiple of $\alpha$ in the last equality, then $\varepsilon$ is replaced by the same multiple of $\varepsilon$. Thus there exists a $K>0$ such that $\varepsilon(\alpha)=K \alpha$. Hence

$$
\begin{gathered}
\max \left\{|\tau-\mu|^{p}+|\tau-\lambda|^{p},|\lambda-\sigma|^{p}+|\mu-\sigma|^{p}\right\} \\
=\max \left\{2|\tau-\eta|^{p}, 2|\eta-\sigma|^{p}\right\}+K .
\end{gathered}
$$

Let $h_{p}^{r}(t)=h_{p}(t)$ if $t>a$ and $h_{p}^{r}(t)=\mu$ if $t \leqslant a$, and define $h_{p}^{\ell}$ similarly, with reversed inequalities. Then each of $h_{p}^{r}$ and $h_{p}^{\ell}$ is continuous at $a$ so, by $[1$, Theorem 5.4] each of $\left|h_{p}^{j}-k\right|^{p}, j=r, l, k=f, g$, is approximately continuous at $a$. By $[1$, Theorem 8.2]

$$
\lim _{\delta \rightarrow 0} \delta^{-1} \int_{a}^{a+\delta}\left|h_{p}^{r}-k\right|^{p}=\left|h_{p}^{r}(a)-k(a)\right|^{p}, \quad k=f, g,
$$

and similar statements hold for $h_{p}^{\ell}$, with integration from $a-\delta$ to $a$. Since $K>0$ there exists a $\delta>0$ such that

$$
\begin{array}{r}
\max \left\{\delta^{-1} \int_{a-\delta}^{a+\delta}\left|h_{p}-f\right|^{p}, \delta^{-1} \int_{a-\delta}^{a+\delta}\left|h_{p}-g\right|^{p}\right\} \\
>\max \left\{\delta^{-1} \int_{a-\delta}^{a+\delta}|\eta-f|^{p}, \delta^{-1} \int_{a-\delta}^{a+\delta}|\eta-g|^{p}\right\} .
\end{array}
$$

If $h_{p}^{*}$ is defined by

$$
h_{p}^{*}= \begin{cases}\eta, & t \in[a-\delta, a+\delta), \\ h_{p}(t), & \text { otherwise, }\end{cases}
$$

then $h_{p}^{*}$ is a better simultaneous $L_{p}$ approximation to $f$ and $g$ than is $h_{p}$.

If $f$ and $g$ are continuous, then they are quasi-continuous and approximately continuous both, so, by (13) and (15), 
Corollary 16. If $f$ and $g$ are continuous, then so is $h_{1}$.

Example (19) in Section 4 shows that not all members of $\mu_{1}(f, g ; \mathcal{M})$ preserve the continuity of $f$ and $g$. As a consequence of (3) and (13) above, we have the following.

Corollary 17. Suppose $p \in[1, \infty)$. If $h_{p} \neq f_{p}$, then $\left\|f-h_{p}\right\|_{p} \leqslant\left\|g-h_{p}\right\|_{p}$. If $f_{p} \neq h_{p} \neq g_{p}$, then $\left\|f-h_{p}\right\|_{p}=\left\|g-h_{p}\right\|_{p}$.

\section{Simultaneous Monotone $L_{1}$-Approximation}

The structure of the set of best simultaneous monotone $L_{1}$ approximations to an arbitrary pair of functions $(f, g)$ is of intrinsic interest. In $[6,7]$, assuming $f=g$, this set was completely characterised in terms of $f$, and in [9], the continuity of the multifunction $f \mapsto \mu_{1}(f ; M)$ was studied. In this section, we present some related results in the context where $f$ and $g$ are not necessarily the same.

LEMMA 18. Let $f$ and $g$ be step functions defined over the same partition of $[0,1]$. Then there exists an element $h \in \mu_{1}(f, g ; \mathcal{M})$ such that $h$ is a step function of the same form as $f$ and $g$.

PROOF: Let $f_{i}$ and $g_{i}$ be the values of $f$ and $g$ on the subinterval $\left(t_{i-1}, t_{i}\right]$. Assume without loss of generality that $g_{i}<f_{i}$. Let $h \in \mu_{1}(f, g ; \mathcal{M})$. If $h$ is not a constant on $\left(t_{i-1}, t_{i}\right]$, then clearly $g_{i} \leqslant h(x) \leqslant f_{i}$ for all $x \in\left(t_{i-1}, t_{i}\right)$, otherwise both of $\|f-h\|_{1}$ and $\|g-h\|_{1}$ can be reduced simultaneously and $h$ would not be an element of $\mu_{1}(f, g ; \mathcal{M})$ any more. Now, we seek a constant $c \in\left[g_{i}, f_{i}\right]$ such that

and

$$
\int_{t_{i-1}}^{t_{i}}\left(f_{i}-h(x)\right) d x=\int_{t_{i-1}}^{t_{i}}\left(f_{i}-c\right) d x
$$

$$
\int_{t_{i-1}}^{t_{i}}\left(h(x)-g_{i}\right) d x=\int_{t_{i-1}}^{t_{i}}\left(c-g_{i}\right) d x .
$$

But it is clear now that $c$ is given by

$$
c=\left(t_{i}-t_{i-1}\right)^{-1} \int_{t_{i-1}}^{t_{i}} h(x) d x .
$$

This completes the proof.

Thus, for any pair of step functions $f$ and $g$, there always exists a step function $h \in \mu_{1}(f, g ; \mathcal{M})$. Clearly, such a step function is not necessarily unique. This will be shown as part of the next example.

In [5], it was shown that the set of best $L_{1}$-approximations to a bounded measurable function $f$ by nondecreasing functions includes its supremum and infimum. However, this is not the case with $\mu_{1}(f, g ; \mathcal{M})$. 
EXAMPLE 19. Take $f \equiv 2$ and $g \equiv 0$ on $[0,1]$. Then any function $h_{c}$ of the form

$$
h_{c}(x)= \begin{cases}c, & 0 \leqslant x \leqslant 1 / 2 \\ 2-c, & 1 / 2<x \leqslant 1\end{cases}
$$

$c \in[0,1]$, is an element of $\mu_{1}(f, g ; \mathcal{M})$, so $\bar{h}:=\sup (f, g ; \mathcal{M}) \geqslant \chi_{[0,1 / 2]}+2 \chi_{(1 / 2,1]}$ and $\underline{h}:=\inf (f, g ; \mathcal{M}) \leqslant \chi_{(1 / 2,1]}$. Thus $d_{1}(f, g ; \bar{h}) \geqslant 3$, so $\bar{h} \notin \mu_{1}(f, g ; \mathcal{M})$. Similarly, $\underline{h} \notin \mu_{1}(f, g ; \mathcal{M})$. Also notice that if $h^{*}(x)=2 x$, then $h^{*} \in \mu_{1}(f, g ; M)$.

This example shows also that the fact that both of $f$ and $g$ are constants doesn't imply that every element of $\mu_{1}(f, g ; \mathcal{M})$ must be also a constant, or even a step function as is the case with $h^{*}(x)=2 x$. It also demonstrates the fact that continuity is not inherited from $f$ and $g$ by all elements of $\mu_{1}(f, g ; \mathcal{M})$.

Next, one might ask about the relation between the set of best $L_{1}$-simultaneous approximations to a pair of functions $f$ and $g$, and the set of best $L_{1}$-approximations to the mean of this pair of functions. In [13], it was shown that $h^{*}$ is the best $L_{2}$ simultaneous approximation to two functions $f$ and $g$ if and only if $h^{*}$ is the best $L_{2}$-approximation to their mean $T=(f+g) / 2$, provided we define $h^{*}$ as the element satisfying

$$
\inf _{h \in \mathcal{M}}\left[\|f-h\|_{2}^{2}+\|g-h\|_{2}^{2}\right]^{1 / 2}=\left(\left\|f-h^{*}\right\|_{2}^{2}+\left\|g-h^{*}\right\|_{2}^{2}\right)^{1 / 2} .
$$

This motivates us to raise a similar question for our case of best $L_{1}$-simultaneous approximation. Is $\mu_{1}(f, g ; \mathcal{M}) \cap \mu_{1}(T ; \mathcal{M}) \neq \emptyset$ for any pair of functions $f$ and $g$; for a special pair of functions, such as continuous functions? How about if $\mu_{1}(T ; \mathcal{M})$ is a singleton? The following example answers these questions.

EXAMPLE 20. Let $f(x)=3-2 x$ and $g(x)=1-4 x$. Then $T(x)=(1 / 2)(f(x)+g(x))=$ $2-3 x$. Clearly $T_{1} \equiv 1 / 2$ is the unique best $L_{1}$-approximation to $T$ by elements of $\mathcal{M}$. However $T_{1} \notin \mu_{1}(f, g ; \mathcal{M})$. Take for example $h^{*} \equiv 29 / 60 \in \mathcal{M}$. Then $d_{1}\left(f, g ; h^{*}\right)<$ $d_{1}\left(f, g ; T_{1}\right)$.

However, the following lemma gives us a condition which guarantees that $\mu_{1}(f, g ; \mathcal{M}) \subseteq \mu_{1}((1 / 2)(f+g) ; \mathcal{M})$.

LEMmA 21. If $d_{1}((1 / 2)(f+g) ; \mathcal{M}) \geqslant d_{1}(f, g ; \mathcal{M})$, then $\mu_{1}(f, g ; \mathcal{M})$ $\subseteq \mu_{1}((1 / 2)(f+g) ; \mathcal{M})$.

Proof: In general, we have for any $h \in \mu_{1}(f, g ; \mathcal{M})$

$$
\begin{aligned}
d^{*}=d_{1}((1 / 2)(f+g) ; M) & \leqslant(1 / 2)\|(f-h)+(g-h)\|_{1} \\
& \leqslant \max \left(\|f-h\|_{1},\|g-h\|_{1}\right)=d_{1}(f, g ; h)=d_{1} .
\end{aligned}
$$


So we obtain equality in the given condition of the theorem. Now, let $h_{1} \in \mu_{1}(f, g ; \mathcal{M})$, and suppose $\left\|f-h_{1}\right\|_{1} \geqslant\left\|g-h_{1}\right\|_{1}$. Then

$$
\begin{aligned}
d_{1}=\left\|f-h_{1}\right\|_{1} & \geqslant(1 / 2)\left(\left\|f-h_{1}\right\|_{1}+\left\|g-h_{1}\right\|_{1}\right) \\
& \geqslant\left\|(1 / 2)(f+g)-h_{1}\right\|_{1} \geqslant d^{*}=d_{1} .
\end{aligned}
$$

Hence $h_{1} \in \mu_{1}((1 / 2)(f+g) ; \mathcal{M})$.

Suppose the hypothesis of (21) holds. Then $\mu_{1}(f, g ; M)=\mu_{1}((1 / 2)(f+g) ; \mathcal{M})$ if $\mu_{1}((1 / 2)(f+g) ; \mathcal{M})$ is a singleton. This occurs when both of $f$ and $g$ are continuous or approximately continuous (see [2]). Even with the assumption of uniqueness of the best $L_{1}$-approximation to the mean $(1 / 2)(f+g)$, the converse of the lemma is still not true in general. The following example illustrates this fact.

EXAMPLE 22. Let $f(x)=x^{2}-1$ on $[-1,1]$ and let $g=-f$. Then

$$
\mu_{1}(f, g ; \mathcal{M})=\mu_{1}((1 / 2)(f+g) ; \mathcal{M})=(1 / 2)(f+g) \equiv 0
$$

However

$$
d^{*}=d_{1}((1 / 2)(f+g) ; \mathcal{M})=0<2 / 3=d_{1}=d_{1}(f, g ; \mathcal{M})
$$

The condition that $d^{*}=d_{1}$ is very vital. To see this, we go back to the two functions $f$ and $g$ given in Example (20) above. There we find that the set $\mu_{1}(f, g ; \mathcal{M})$ consists of a single element, namely $h_{1} \equiv 2 \sqrt{3}-3$. However

$$
\begin{aligned}
d_{1}=\left\|f-h_{1}\right\|_{1} & =\left\|g-h_{1}\right\|_{1}=5-2 \sqrt{3} \\
& >3 / 4=d^{*}=d_{1}\left((1 / 2)(f+g) ; h^{*}\right),
\end{aligned}
$$

where $h^{*} \equiv 1 / 2$ is the unique best $L_{1}$-approximation to $(1 / 2)(f+g)$.

\section{References}

[1] A.M. Bruckner, Differentiation of Real Functions: Lecture notes in mathematics 659 (Springer-Verlag, Berlin, Heidelberg, New York, 1978).

[2] R.B. Darst and R. Huotari, 'Best $L_{1}$-approximation of bounded approximately continuous functions on $[0,1]$ by nondecreasing functions', J. Approx. Theory 43 (1985), 178-189.

[3] D.S. Goel, A.S.B. Holland, C. Nasim and B.N. Sahney, 'On best simultaneous approximation in normed linear spaces', Canad. Math. Bull. 17 (1974), 523-527.

[4] D.S. Goel, A.S.B. Holland, C. Nasim and B.N. Sahney, 'Characterization of an element of best $\ell^{p}$-simultaneous approximation', $S$. Ramanaujan Memorial Volume, Madras (1984), 10-14.

[5] R. Huotari and D. Legg, 'Best monotone approximation in $L_{1}[0,1]$ ', Proc. Amer. Math. Soc. 94 (1985), 279-282. 
[6] R. Huotari, D. Legg, A. Meyerowitz and D. Townsend, 'The natural best $L_{1}$-approximation by nondecreasing functions', J. Approx. Theory 52 (1988), 132-140.

[7] R. Huotari, A. Meyerowitz and M. Sheard, 'Best monotone approximants in $L_{1}[0,1]$ ', $J$. Approx. Theory 47 (1986), 85-91.

[8] R. Huotari and S. Sahab, 'Simultaneous monotone $L_{p}$ approximation, $p \rightarrow \infty$ ', Canad. Math. Bull. (to appear).

[9] D. Legg and D. Townsend, 'Sets of best $L_{1}$ approximants', J. Approx. Theory 59 (1989), 316-320.

[10] D. Landers and L. Rogge, 'Natural choice of $L_{1}$ approximants', J. Approx. Theory 33 (1981), 268-280.

[11] D. Landers and L. Rogge, 'On projections and monotony in $L_{p}$-spaces', Manuscripta Math. 26 (1979), 363-369.

[12] I. P. Natanson, Theory of functions of a real variable (Ungar, New York, 1955).

[13] G.M. Philips and B.N. Sahney, 'Best simultaneous approximation in the $L_{1}$ and $L_{2}$ norms', in Theory of approximation with applications, Editors A.G. Law and B.N. Sahney (Academic Press, New York, 1976).

[14] M.J.D. Powell, Approximation theory and methods (Cambridge, 1981).

[15] S. Sahab On the monotone simultaneous approximation on $[0,1]$, Bull. Austral. Math. Soc. 39 (1988), 401-411.

[16] S. Sahab, 'Natural choice of best $L_{1}$-simultaneous approximants', Tamkang J. Math. 20 (1989), 147-157.

[17] S. Sahab, 'Best simultaneous approximation of quasicontinuous functions by monotone functions', J. Austral. Math. Soc. (Ser. A) (to appear).

[18] I. Singer, Best approximation in normed linear spaces (Springer Verlag, Berlin, Heidelberg, New York, 1970).

[19] A.C.M. Van Rooij and W.H. Schikhof, A second course on real functions (Cambridge, 1982).

Department of Mathematics

Idaho State University

Pocatello, ID 83209

United States of America
Mathematics Department

King Abdulaziz University

Jeddah 21413

Saudi Arabia 